\title{
A Comparative Study of Image Enhancement Techniques
}

\author{
Swati Khidse \\ Research Student \\ Government College of Engineering, Station Road, \\ Osmanpura, Aurangabad, Maharashtra, India
}

\author{
Meghana Nagori \\ Assistant Professor CSE Dept. \\ Government College of Engineering, Station Road, \\ Osmanpura, Aurangabad, Maharashtra, India
}

\begin{abstract}
Images are the main source of acquiring information from the real world. Images are captured or taken in different situations and position. So there is need for image preprocessing of the captured image for the purpose of removal of noise, unwanted background, making sharp or blur according to application. Image enhancement is the first preprocessing step in image processing, with the help of which the authors propose the image with more clarity. In this paper the authors describes various techniques of image enhancement and compare it with image fusion techniques, with the help of various error analysis techniques. Image fusion techniques are assessed using the various metrics. A comparative study is carried out on different categories of images.
\end{abstract}

\section{General Terms}

Digital image processing, Image enhancement

\section{Keywords}

Image enhancement, Image fusion, metrics, Error Analysis

\section{INTRODUCTION}

Image enhancement refers to the sharpening of image features such as edges, boundaries, or contrast to make images clearer understandable than the original image. Image enhancement is applied in different field where images have to be analyzed for e.g. medical image analysis, analysis of images from satellites etc. After enhancing, image can be used for further analysis, detection, segmentation and recognition in image processing. Real world images are acquired with low contrast and unsuitable for human eyes to read, such as medical and industrial X-ray images, so to have a clear perception of the image it is necessary to enhance the image. Image enhancement can be classified into two broad categories: spatial domain and frequency domain. Spatial domain technique operates directly on pixels whereas frequency domain operates on the Fourier transform of an image. The spatial domain technique is having different advantages like it is easy to understand and study, complexity is also less, thus it can be used in real time applications, but on the other hand it has some drawbacks like it does not provide adequate robustness. Frequency domain image enhancement technique basically works by manipulating Fourier coefficients. This method describes mathematical functions with respect to the frequency and it operates on Frequency transform i.e. Fourier coefficients discrete wavelet transform (DWT), and discrete cosine transform (DCT). The advantages are it is easy for computation complexity and robust. Similarly it has having some drawbacks like it cannot able to enhance all the parts of the image simultaneously very well, difficult to automate the procedure of image enhancement.
Consider the below Figure 1 showing the effects before and after image enhancement. In this we have consider only gray scale image but same method applies to color images also.
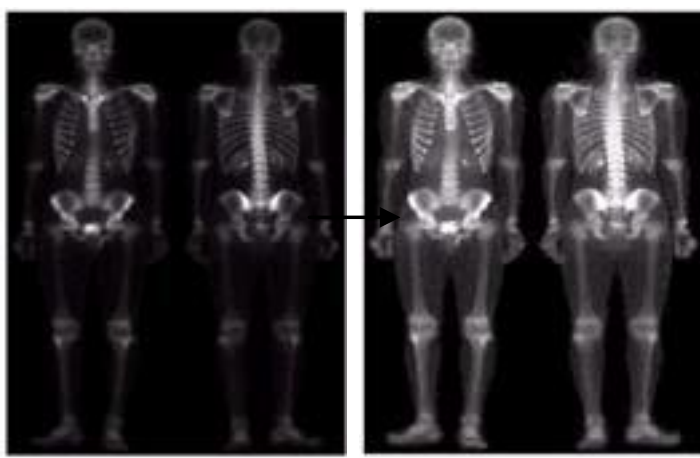

Figure 1: Result before $\&$ after image enhancement

In practical forensic examinations and in many fields, we cannot obtain all information from just one image; we need information from images with difference light sources. Image fusion is a process of combining two or more images into an image and provides more information than one image; these images may come from one sensor or multiple sensors. For e.g. in medical applications, the fusion of computer tomography (CT) and magnetic resonance images (MRI) may display the whole structure in bioscience, in flight navigation, the fusion of visual and infrared images can aid pilots navigate in poor weather conditions; in military application, it also assist armed forces, where the enemies are in the night fighting. The advantages of image fusion are a) Improve reliability (having redundant information), b) Improve capability (having complementary information).

Image fusion can be classified into two domain types a) Spatial domain, b) Transform domain. In spatial domain there are different techniques like Averaging, Select maxima, Select minima and etc. It basically works directly on the image pixels. The pixel values of the image are manipulated. In frequency domain methods the image is first transferred in to frequency domain. Firstly, Fourier Transform of the image is computed. All the Fusion operations are performed on the Fourier transform of the image and then the Inverse Fourier transform is performed to get the resultant image. The remaining section of the paper is divided into Literature survey, proposed system work, Performance evaluation, and Error analysis techniques respectively.

\section{LITERATURE SURVEY}

The basic aim of the image enhancement is to manipulate the attributes of the image to make it more suitable for a given task. Much work has been done in the field of the image 
enhancement using spatial domain and frequency domain techniques. In spatial domain techniques like logarithmic transforms, histogram equalization, power law transforms these all are based on the direct manipulation of the pixels in the image. These are useful for directly altering the gray level values of individual pixels and thus the overall contrast of the complete image [1].
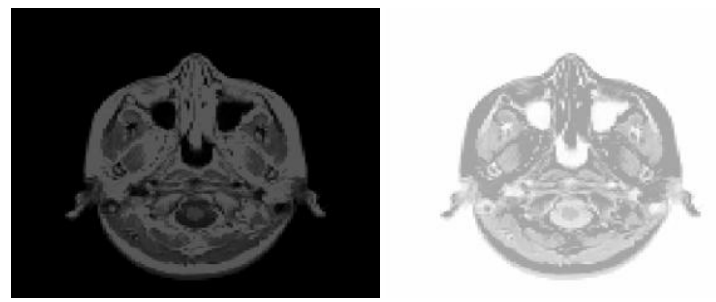

Figure 2: Original MRI image \& Image Negative
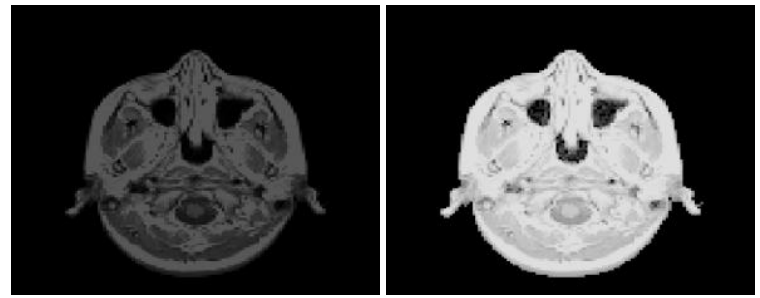

Figure 3: Original MRI image \& Log transform of image

In frequency domain image enhancement, different kinds of filters are used for smoothing of the images or for sharpening of the images [1].Basically these are used in sharpness enhancement of stereo images.

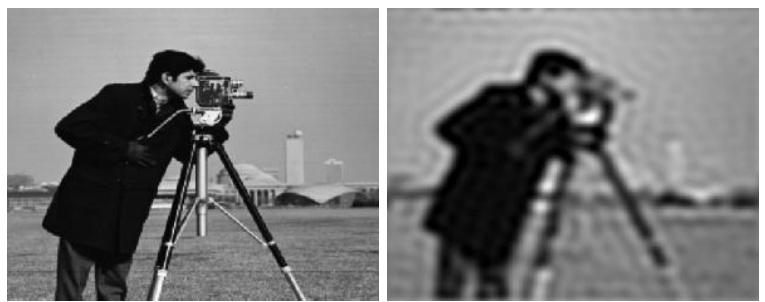

Figure 4: Original image \& Ideal Low pass Filter
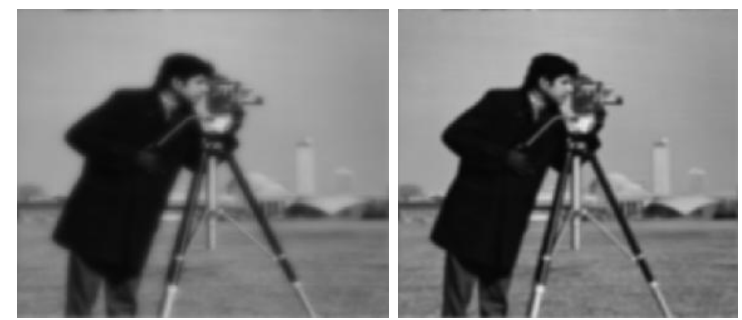

Figure 5: Butter worth Low Pass Filter \& Gaussian Low Pass Filter

The work done by various researchers in the field of image enhancement are discussed here, [2] Mussarat Yasmin, Muhammad Sharif, Saleha Masood, Mudassar Raza and Sajjad Mohsin, "Brain Image Enhancement - A Survey, 2011" The basic aim of image enhancement is to analyze the brain images precisely in order to effectively diagnose and examine the diseases and problems. It has provided a very efficient way to deal with the detail study and examination of brain.

A. Poljicak, L. Mandic, M. Strgar Kurecic, "Improvement of the Watermark Detector Performance Using Image Enhancement Filters,"2012" [3] considered the influence of some digital image processing techniques on the watermark detection rate. Watermarking methods are very much sensitive to complex degradation attacks like JPEG compression, or print scan process due to impact of which the detection rate of a watermark method decreases considerably after such attacks on a watermarked image. By reducing the degradation of the image by using Unsharp, Laplacian or deconvolution filters the detection rate can be improved for watermarking methods.

Xiaoying Fang, Jingao Liu, Wenquan Gu, Yiwen Tang ,“ A Method to Improve the Image Enhancement Result based on Image Fusion,"2011 "[4] proposes a method for the improvement of the image enhancement results with the image fusion technique with evaluation on sharpness.

Local contrast enhancement attempts to increase the appearance of light-dark transitions. It is like optimization problem. Local contrast enhancement attempts to increase "local" contrast in the smaller regions of the image and at the same time preventing increase in the "global" contrast of the image. Image enhancement techniques provide a greater range of enhancement for the images in various application domains, but it does not provide an efficient result in situation: When images are captured from different angles and directions, in this case only one image cannot provide complete information of the image. So there is need to fuse these different images and then use an image fusion technique over them for better image enhancement. Image fusion [5] is used in various application domains and it is also providing very good results. This technique is used in [6] Forensic applications (footprint and money texture), medical diagnosis (detection of tumor or any defect inspection) the fusion of the MRI and the CT scan image of the brain provide more clear visual information than the input image, remote sensing, military surveillance.

\section{PROPOSED SYSTEM WORK}

The flow of the proposed system design will be as shown in the algorithm.

Algorithm

1. Take input image

2. Perform image enhancement with spatial domain \& frequency domain.

3. Perform image fusion with spatial domain \& frequency domain.

4. Objective assessment of image fusion techniques with different metrics.

5. Comparisons of step 2 and step 3 based on error analysis techniques.

The Algorithm describes the procedure for carrying out the system work. The authors propose their research work with various techniques \& methods to carry out the image enhancement techniques in tabular format. In Table 1 the various [5], [7] techniques are specified in image enhancement domain and in image fusion. 
Table 1: Image enhancement $\&$ fusion methods

\begin{tabular}{|c|c|c|c|}
\hline Sr.no & $\begin{array}{l}\text { Type of } \\
\text { images }\end{array}$ & Image enhancement & Image fusion \\
\hline$[1]$ & $\begin{array}{l}\text { Satellite } \\
\text { image } \\
\text { And } \\
\text { etc. }\end{array}$ & 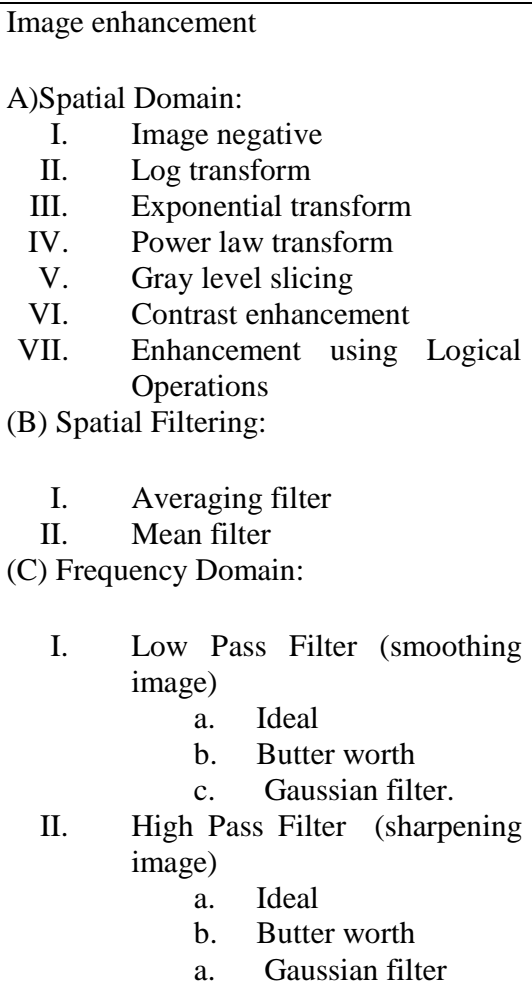 & 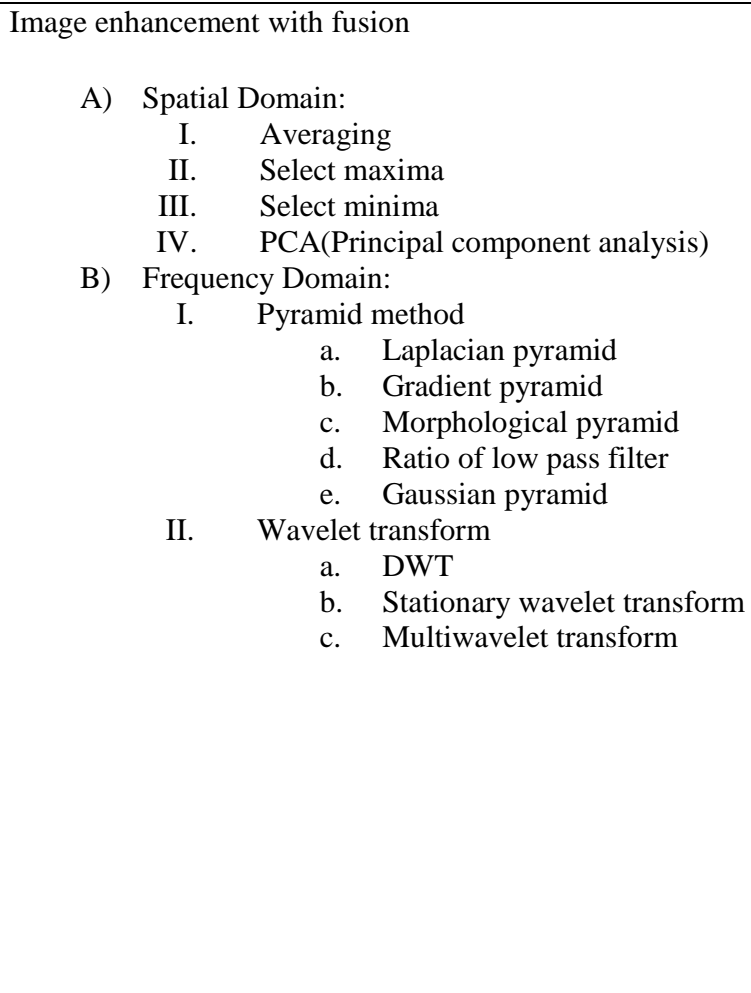 \\
\hline
\end{tabular}

Various metrics [8] have been used for objective assessment of the image enhancement techniques. With this the authors are comparing the different image enhancement fusion techniques:

\subsection{Information theory based metrics:}

4.1.1 Normalized Mutual Information $\left(Q_{M I}\right)$ : It represents mutual dependence of two variables. Mutual information is represented by MI (A, F), entropy with $\mathrm{H}(\mathrm{A})$.

$$
Q_{M I}=2\left[\frac{M I(A, F)}{H(A)+H(F)}+\frac{M I(B, F)}{H(B)+H(F)}\right]
$$

\subsubsection{Fusion Metric-Based on Tsallis Entropy}

$\left(Q_{T E}\right)$ : Tsallis entropy represents the divergence measure of degree of dependence between two variables.

$$
Q_{T E}=\frac{I^{q}(A, F)+I^{q}(B, F)}{H^{q}(A)+H^{q}(B)-I^{q}(A, B)}
$$

\subsubsection{Nonlinear Correlation Information Entropy}

$\left(Q_{N C I E}\right)$ : The Eigen value of the nonlinear correlation matrix is $\lambda_{i}$ for $(\mathrm{i}=0,1,2,3)$.
4.2 Image feature based metrics: Assessment is implemented by measuring how the features are transferred from the input images to the fused one.

4.2.1 Image Fusion Metric-Based on Spatial Frequency $\left(Q_{S F}\right)$ : Spatial Frequency is used to measure the activity level of an image $\mathrm{I}(\mathrm{x}, \mathrm{y})$.

$$
\begin{array}{r}
S F=\sqrt{(R F)^{2}+(C F)^{2}+(S D F)^{2}}, \\
Q_{S F}=\left(S F_{F}-S F_{R}\right) / S F_{R}
\end{array}
$$

RF, CF, SDF, and MDF are the four first-order gradients along the four directions.

\subsubsection{Image Fusion Metric-Based on Phase}

Congruency $\left(Q_{P}\right)$ : Phase congruency, provides an absolute measure of image feature. The metric is defined as a product of three correlation coefficients.

$$
Q_{P}=\left(P_{p}\right)^{\alpha}\left(P_{M}\right)^{\beta}\left(P_{m}\right)^{\gamma}
$$

$\mathrm{p}, \mathrm{M}, \mathrm{m}$ refers phase congruency (p), maximum, and minimum moments, respectively.

$$
\begin{aligned}
& P_{P}=\max \left(C_{A F}^{p}, C_{B F}^{p}, C_{S F}^{p}\right), \\
& P_{M}=\max \left(C_{A F}^{M}, C_{B F}^{M}, C_{S F}^{M}\right),
\end{aligned}
$$


$P_{m}=\max \left(C_{A F}^{m}, C_{B F}^{m}, C_{S F}^{m}\right)$,

$C_{x y}^{k},\{k \mid p, M, m\}$ stands for the correlation coefficients between two sets $\mathrm{x}$ and $\mathrm{y}$.

\subsection{Information theory based metrics:}

4.1.1 Normalized Mutual Information $\left(Q_{M I}\right)$ : It represents mutual dependence of two variables. Mutual information is represented by MI (A, F), entropy with $\mathrm{H}(\mathrm{A})$.

$$
Q_{M I}=2\left[\frac{M I(A, F)}{H(A)+H(F)}+\frac{M I(B, F)}{H(B)+H(F)}\right]
$$

\subsection{Image structural similarity based}

metrics: A structural similarity index measure (SSIM) for images $\mathrm{A}$ and $\mathrm{B}$ defined as

$$
\begin{gathered}
\operatorname{SSIM}(A, B)=[l(A, B)]^{\alpha}[c(A, B)]^{\beta}[s(A, B)]^{\gamma} \\
=\left(\frac{2 \mu_{A} \mu_{B}+C_{1}}{\mu_{A}^{2}+\mu_{B}^{2}+C_{1}}\right)^{\alpha}\left(\frac{2 \sigma_{A} \sigma_{B}+C_{2}}{\sigma_{A}^{2}+\sigma_{B}^{2}+C_{2}}\right)^{\beta}\left(\frac{\sigma_{A B}+C_{3}}{\sigma_{A}+\sigma_{B}+C_{3}}\right)^{\gamma}
\end{gathered}
$$

In this $\mu_{A}$ and $\mu_{B}$ are the average values of images $\mathrm{A}(\mathrm{i}, \mathrm{j})$ and $\mathrm{B}(\mathrm{i}, \mathrm{j}), \sigma_{A}, \sigma_{B}$, and $\sigma_{A B}$ are the variance and covariance. The 1 $(\mathrm{A}, \mathrm{B}), \mathrm{c}(\mathrm{A}, \mathrm{B})$ and $\mathrm{s}(\mathrm{A}, \mathrm{B})$ are the luminance, contrast, and correlation components, resp.

\section{ERROR ANALYSIS TECHNIQUES}

Error analysis techniques [5], [9] can be used for comparison of different image enhancement techniques:

\subsection{Normalized Mean Square Error}

Method (NMSE): The NMSE compares the mean of a series against the predicted values. If the NMSE value is greater than 1, then the predictions are going worse than the series mean and vice versa. The following formula is used to calculate NMSE:

$$
N M S E=\frac{\sum_{i=-N / 2}^{N / 2} \sum_{j=-N / 2}^{N / 2}[f(i, j)-g(i, j)]^{2}}{\sum_{i=-N / 2}^{N / 2} \sum_{j=-N / 2}^{N / 2} f(i, j)^{2}}
$$

Where $f(i, j)$ is the original image with size $\mathrm{N}^{*} \mathrm{~N}$, and $g(i, j)$ is the filtered image with size $\mathrm{N}^{*} \mathrm{~N}$.

\subsection{Projected Mean Square Error Method}

(PMSE): PMSE used to study the characteristic of the error overall the filtered image and is calculated using the following formula:

$$
\operatorname{PMSE}=\frac{\sum_{j=-N / 2}^{N / 2}[f(i, j)-g(i, j)]^{2}}{\sum_{i=-N / 2}^{N / 2} \sum_{j=-N / 2}^{N / 2} f(i, j)^{2}}
$$

5.3 Normalized Absolute Error (NAE): NAE is the average of the absolute difference between the originally and the enhanced image divided by the original image. The large the value of NAE the poor is the image.

$$
N A E=\frac{\sum_{i=1}^{m} \sum_{j=1}^{n}\left(\left|A_{i j}-B_{i j}\right|\right)}{\sum_{i=1}^{m} \sum_{j=1}^{n}\left(A_{i j}\right)}
$$

5.4 Maximum Difference (MD): It represents the maximum difference between two images.

$$
M D=\max \left(\left|A_{i j}-B_{i j}\right|\right), i=1,2, \ldots m ; j=1,2, \ldots n
$$

5.5 Average Difference: This measure shows the average difference between the pixel values given by

$$
A D=\frac{1}{m n} \sum_{i=1}^{m} \sum_{j=1}^{n}\left(\left|A_{i j}-B_{i j}\right|\right)
$$

5.6 Normalized Cross-Correlation (NCC): Normalized cross correlation are used to find out similarities between fused image and registered image is given by

$$
N C C=\frac{\sum_{i=1}^{m} \sum_{j=1}^{n}\left(A_{i j} * B_{i j}\right)}{\sum_{i=1}^{m} \sum_{j=1}^{n}\left(A_{i j}\right)^{2}}
$$

5.7 Structural Content (SC): It is correlation based measure for the original and enhanced image.

$$
S C=\frac{\sum_{i=1}^{m} \sum_{j=1}^{n}\left(A_{i j}\right)^{2}}{\sum_{i=1}^{m} \sum_{j=1}^{n}\left(B_{i j}\right)^{2}}
$$

\section{CONCLUSION}

In this paper of comparative study, with image set over the different image enhancement techniques, authors will be able to predict the performance of the particular technique over its counterparts. In case of MRI image of brain by using either spatial or frequency domain image enhancement techniques good results are not obtained as compare to the image fusion technique in which the MRI image is fused with the CT image to get the resultant more enhanced image. Based on the obtained good results, the particular method can also be used in videos, images captured by low resolution cameras, scanned copies of various documents, etc. for image enhancement.

\section{ACKNOWLEDGEMENTS}

The authors express gratitude to Principal, Head of Department (CSE) Prof. V. P. Kshirsagar, Government College of Engineering, Station Road, Aurangabad, and Maharashtra India. They also express their sincere thanks all the faculty members of CSE Department Government College of Engineering, Station Road, Osmanpura, Aurangabad, and Maharashtra, India for their constant support and enthusiasm.

\section{REFERENCES}

[1] Atul Bansal, Rochak Bajpai, J. P. Saini, "Simulation of Image Enhancement Techniques Using Matlab", IEEE 2007.

[2] M. Yasmin, M. Sharif, S. Masood, M. Raza and S. Mohsin, "Brain Image Enhancement - A Survey", World Applied Sciences Journal 17 (9): 1192-1204, 2012, ISSN 1818-4952@ IDOSI Publications, 2012.

[3] A. Poljicak, L. Mandic, M. Strgar Kurecic,“ Improvement of the Watermark Detector Performance Using Image Enhancement Filters," IWSSIP 2012, 11-13 April 2012, Vienna, Austria. 
[4] X. Fang, J. Liu, W. Gu, Y. Tang, “ A Method to Improve the Image Enhancement Result based on Image Fusion", 978-1-61284-774-0/11 @2011 IEEE.

[5] Shivsubramani Krishnamoorthy, K. P. Soman, "Implementation and Comparative Study of Image Fusion Algorithms", IJCA 2010, pp.25-35, pp29-30.

[6] C.Y. Wen, J.K. Chen, "Multi-resolution image fusion technique and its application to forensic science", 2004 Elsevier Ireland Ltd, pp.229-231.

[7] Rafael, C. Gonzalez and R. E. Woods, Digital image processing: 2nd edition, Prentice Hall, 2002.
[8] Zheng Liu, Erik Blasch, Zhiyun Xue, Jiying Zhao, Robert Laganie, Wei Wu, "Objective Assessment of Multiresolution image fusion Algorithms for Context Enhancement in Night Vision: A Comparative Study", IEEE transactions on pattern analysis and machine intelligence, vol. 34, no. 1, January 2012, pp.95-98.

[9] Moustafa Abdel Aziem Moustaf, "Quntitative and qualitative evaluations of image enhancement techniques", IEEE 2004, pp.665-666. 